\title{
The effect of varying linear perspective, movement parallax, and speed of rotation on perceived oscillation of two-dimensional trapezoidal stimuli
}

\author{
JANE H. BRADEN \\ Fairleigh Dickinson University, Teaneck, New Jersey 07666
}

\begin{abstract}
Two experiments were conducted using two-dimensional screen projections of trapezoidal stimuli in an investigation of the role of the cues of linear perspective and monocular movement parallax in producing apparent oscillation of the Ames (1951) trapezoidal window. The first experiment was designed to determine the effect of covarying linear perspective and movement parallax on number of perceived oscillations of the stimulus figures. Ten stimuli, ranging from a rectangle to an extreme trapezoid, and four projection values, three different polar projections, and a parallel projection condition were employed. The data supported the hypotheses that: (1) the number of perceived oscillations is dependent upon the relative saliency of the two cues in the stimulus display; and (2) the amount of intersubject variability increases as the ambiguity of the stimulus display increases. In the second experiment, speed of rotation of the same stimuli was varied under both polar and parallel projection conditions. The data indicated that a decrease in the number of perceived oscillations at higher rotational speeds cannot be attributed to an increase in the effectiveness of movement parallax.
\end{abstract}

Normally, visual cues cooperate to enable the observer to obtain veridical information about the environment. In the case of perceptual conflict, Ittleson (1960) has indicated four possible resolutions, one of which is alternation over time, with one or the other cue dominating at a particular time. In the Ames (1951) trapezoidal window illusion, the above solution seems to occur: sometimes continuous rotation is perceived, sometimes change of direction (oscillation) is perceived. The resolution of perceptual conflict at a particular time ought to depend on the relative saliency of the conflicting cues. According to Graham's (1963) analysis of the Ames window, perceived oscillation is caused by two factors: ambiguity of the cue of movement parallax with regard to direction of rotation, and resolution of this ambiguity by means of the cue of linear perspective. "Linear perspective" refers to the fact that a constant distance between points subtends a smaller and smaller visual angle as the distance from the eye to these points is increased. Thus, a figure that is seen by an observer as rectangular in the frontal plane of view will be perceived as trapezoidal

The preparation of this manuscript was supported in part by the PHS Research Grant 501 EY 00391 from the National Eye Institute and also in part by Contract N00014-67-A-0108-0009 between Columbia University and the Office of Naval Research. The author wishes to express gratitude to the late Dr. Clarence Graham and to Dr. Barbara (Gillam) Lawergren for their help in conducting this research. in shape when rotated either clockwise or counterclockwise about its vertical axis at an angle to the observer, since one vertical side will now be further away from the observer and the other side will be closer to the observer. In the Ames window, the cue of linear perspective is produced by the relative lengths of the long and short vertical sides of the trapezoid, as well as by the painted shadows and "panes."

If a stimulus is static, no information exists as to its true orientation to the observer. For example, under conditions of static slant, a rectangle may be considered a slanted rectangle or a frontoparallel trapezoid. However, dynamic stimulus arrays (i.e., translation or rotation of physical stimuli) do give information about those stimuli, given the assumption of rigidity of the distal stimulus. "Movement parallax" refers to the differential angular velocity on the retina which exists between any pair of points on a moving object and the line of sight to the observer whenever that object changes its orientation in space. Ordinarily, given a rigid distal stimulus subtending a sufficiently large visual angle (Gillam, 1969), rotation under polar lighting conditions involves both vertical and horizontal expansion and contraction of the proximal stimulus (movement parallax) which serves to inform the observer as to the direction of motion (Hershberger, 1967).

One of the cues involved in the Ames illusion, movement parallax, gives the observer correct information about the direction of rotation provided 
this cue is not ambiguous. However, the linear perspective cue provides incorrect information, i.e., that the longer side of the figure is closer to the observer. Therefore, when the long vertical side is actually behind the short vertical side as the window is rotated, movement parallax is in conflict with linear perspective. Graham (1963) argues that, when movement parallax is ambiguous, the cue of linear perspective is used to resolve the ambiguity of direction of rotation, and the figure appears to oscillate, with the long side perceived as always in front of the frontal plane. From Graham's theory, several predictions can be made: (1) If movement parallax is ambiguous and linear perspective strong, oscillation of the stimulus figure should occur more frequently; (2) if linear perspective is weak and movement parallax strong, continuous rotation should be more often perceived; and (3) conflict should be greatest when both cues are either weak or strong. The purpose of Experiment 1 was to test the above hypotheses under conditions where the relative strengths of both cues were covaried.

In the present experiments, linear perspective was manipulated through the use of opaque metal figures ranging from rectangular to the most extreme trapezoid. No "panes" were cut out, since it has been found (Zegers, 1965), using planar objects rotating in depth, that there is no difference between trapezoids designed to look like windows and plain trapezoids in number of perceived oscillations.

The strength of movement parallax may be decreased in either of two ways: (1) by increasing the distance between an observer and a rotating stimulus; and (2) by increasing the distance of a light source from a screen onto which the stimulus is projected. Movement parallax may be made ambiguous by sufficiently decreasing the visual angle (Method 1), or, alternatively, by projecting the object under parallel lighting conditions (Method 2). For the second method, a collimating lens may be employed. In either case, horizontal and vertical expansion and contraction are the same for clockwise and counterclockwise rotation. The second method was chosen for the present experiments. The parallel lighting condition was produced by the use of a collimating lens, and three different polar lighting conditions, resulting in three different values of movement parallax, were used. Polar projections were produced by removing the collimating lens and varying the distance between the light source and the screen.

Much of the previous experimental work on the Ames window has employed viewing conditions described by Method 1, similar to those of Zegers and Murray (Note 1). In this case, a luminescent trapezoid is presented to the subject in a darkened room, and the subject views it at different distances. With large enough visual angles, sufficient move- ment parallax will enable the subject to differentiate between clockwise and counterclockwise movement. With smaller visual angles, the saliency of movement parallax will be cut down and, as Zegers and Murray found, proportionately fewer continuous rotations and a greater number of oscillations will be perceived. The use of rotating stimuli between a light source and screen, as employed in the present experiments, is a technique which gives rise to the kinetic depth effect (KDE), a term first used by Wallach and O'Connell (1953), although the phenomenon was first demonstrated by Metzer (1934). The term "KDE" is used to describe the transformation of two-dimensional stimuli into apparently three-dimensional stimuli when the stimuli are in motion. Wallach and O'Connell (1953) found that, in order for the KDE to occur, changes in both direction and apparent size of the stimulus must be present. Both of these cues were evident in the stimulus display used in the present experiments. The KDE may be explained by Gibson's (1966) analysis of motion perception as a series of perspective transformations of objects which provide information to the observer. An alternative theory, presented by Braunstein (1962), involves the identification of retinal events as cues to depth. Vector analysis of motion and depth perception as it relates to the KDE has been done by Borjesson and von Hofsten (1972) and by Johanssen (1973). It should be noted that the use of a projection screen technique with polar lighting conditions results in changes opposite to those which take place when the object is viewed by the eye, since these changes now depend on the object's distance from the light source, not from the eyes. With normal viewing, movement parallax changes are greater in front of the frontal plane of sight; with a screen, the changes are greater in back of the frontal plane, i.e., nearer the light source. However, the saliency of movement parallax may be successfully manipulated by the uses of an object rotating in depth or a two-dimensional screen projection.

Previous research (Freeman \& Pasnak, 1968; Zegers \& Murray, Note 1) using objects rotating in depth, has indicated that the amount of linear perspective, specified either by the ratio of the two vertical sides or by the amount of slope of the horizontal lines of the figure, will affect the number of perceived reversals of the Ames window. Cross (1969) found that by increasing the ratio of two unconnected vertical lines rotated about a single axis, the number of perceived oscillations increased. Apparent oscillation of rotating figures is, however, not found only with trapezoidal stimuli or unequallength vertical lines. Cross and Cross (1969), Day and Power (1965), and Mulholland (1956) have found that apparent reversals of other shapes, such as ellipses 
and circles, also occur. Day and Power (1965) attribute all reversals to an identity of retinal motion characteristics for clockwise and counterclockwise motion, and postulate that apparent changes in direction of rotation occur on a random basis. Their argument as to identical retinal motion may be true when movement parallax is ambiguous, but cannot hold under the conditions they employed. Gillam (1969) notes that the figures used by Day and Power subtended visual angles of about $10^{\circ}$; she estimates, from Zeger's (1965) data, that such figures must be $5^{\circ}$ or less for them to be ambiguous as to direction of rotation. Mulholland (1956) and Pastore (1952) report apparent oscillation of rectangular stimuli provided the figures were below $10^{\circ}$ of visual angle. Furthermore, Cook, Mefferd, and Wieland (1967) produced evidence that apparent reversal is not random, but occurs sytematically.

That other figures besides trapezoids may reverse direction does not contradict Graham's (1963) hypothesis as to the causes of the Ames illusion. Ames himself found that apparent reversals occurred with other forms, and states that the trapezoid was selected for study because of the lower variability in amount and stability of the illusion with this shape (Kilpatrick, 1953). It has also been shown by Cahill (1969) that trapezoids oscillate more frequently than rectangles.

Investigators have sought to isolate the important cues to direction of rotation using various shapes (Power, 1967) and luminescent wire figures (Murch, $1970)$ rotating in space, which contained either or both vertical and horizontal cues to motion. Both authors conclude that changes in the horizontal component of the stimuli is an important cue to direction of rotation; however, the data obtained by Murch also indicate that there is an interaction between vertical and horizontal components of stimuli which enables an observer to obtain information about direction of rotation.

Several experiments have been performed using two-dimensional screen projections of rotating stimuli. Graham and Gillam (1970) showed that a trapezoidal silhouette projected on a screen in parallel projection reversed direction twice per revolution (oscillated $100 \%$ of the time) for all subjects, as Graham's (1963) theory predicted. In addition, subjects reported that they perceived the long vertical side of the figure as always in front of the frontoparallel plane, regardless of its true position. It was concluded by these authors that subjects utilized the linear perspective cue (the difference in length between the long and short vertical sides) to resolve ambiguity of rotational direction.

Braunstein and Payne (1967) used computergenerated stimulus projections and found that, in parallel projection, a trapezoid osciilated $100 \%$ of the time, while a rectangle oscillated only $50 \%$ of the time. As the degree of polarity of projection was increased, the rectangle was never perceived as oscillating, while the trapezoid oscillated progressively less frequently. In their discussion, they state: "The simplest explanation, somewhat related to Graham's, is that the subject resorts to an alternative, and possible inappropriate, source of information, when motion-perspective is ambiguous. A probable secondary source is the relative size of the two vertical sides of the trapezoid" (p. 403).

The main purpose of Experiment 1 was to investigate the frequency of perceived oscillation and continuous rotation of the Ames window under conditions where both cues, linear perspective and movement parallax, are of several different relative strengths. It was hypothesized that, when linear perspective was strong and movement parallax weak, oscillation should predominate; rotation should predominate when the relative strength of the two cues was reversed.

Zegers and Murray (Note 1) found a high degree of intersubject variability under all conditions of their experiment, leading them to conclude that the effects of central organizing processes upon perception are primary. They state, "Wide individual differences suggest that the weight given to different cues varies among people" (p. 116). Graham and Gillam (1970) found no variance in number of perceived oscillations when the cue of movement parallax was made ambiguous by the use of parallel projection, leaving the linear perspective cue to be utilized by subjects. A second purpose of Experiment 1 was to determine how intersubject variability would be affected by varying the strengths of the cues of linear perspective and movement parallax. It was predicted that, in the case of perceptual conflict, variability would be high; however, when one or the other cue was dominant, variability between subjects would decrease.

Experiment 2 was conducted to determine the effect of speed of rotation upon frequency of perceived oscillation. Borjesson (1971) found that an increase in the speed of oscilloscope-generated outlines of trapezoids from $3 \mathrm{rpm}$ to $30 \mathrm{rpm}$ led to a decrease in frequency of perceived oscillation. This may imply that movement parallax cues are better utilized at higher speeds of rotation. Borjesson (1971) states, "It is a necessary condition for apparent oscillation that the changes associated with a certain direction of rotation are not utilized by the visual system. This can be accomplished by decreasing the amount or rate of these changes" (p. 305). In order to test this hypothesis, speed of rotation was varied using both polar and parallel lighting conditions in Experiment 2 .

It was felt that, if a decrease in number of per- 
ceived oscillations at higher rotational speeds was found when movement parallax was ambiguous (parallel lighting condition) then this decrease could not be attributed to an increase in the saliency of this cue.

\section{EXPERIMENT 1}

The goal of the first experiment was to determine the effect of systematically covarying the saliency of the cues of linear perspective and movement parallax on perceived oscillation of screen-projected stimuli. Linear perspective was manipulated by the difference in length between the vertical sides of the stimuli. Movement parallax $(\varphi)$ was changed in strength by using a collimating lens in the parallel projection condition and varying the distance of the light source and stimulus figures from the screen in the polar projection condition.

\section{Method}

Four separate experimental conditions were run, with a different value of $\varphi$ for each condition, and one control condition was run with a $\varphi$ value similar to that of one of the experimental conditions. $\varphi$ was defined as the number of degrees per second change of visual angle of the short vertical side of the figures between $45^{\circ}$ toward the subject and $45^{\circ}$ away from the subject. It should be noted that $\varphi$ could also have been specified by the change in the horizontal component of the stimuli. The same 10 stimulus figures were used for all conditions. Speed of rotation was constant at $5 \mathrm{rpm}$ for all conditions.

Subjects. Ten different subjects participated in each of the five conditions.

Apparatus. Ten opaque metal figures were employed as stimuli. The length of the short vertical sides was $25.25 \mathrm{~mm}$; their width was $37.5 \mathrm{~mm}$. The axis of rotation was placed at $17.5 \mathrm{~mm}$ from the short side of the figure. The strength of linear perspective was manipulated by varying the lengths of the long vertical sides of the figures. Table 1 shows the lengths (millimeters) of the long vertical side for each stimulus. The apparatus used was similar to that employed by Graham and Gillam (1970). A point source of light illuminated the stimulus figures onto a groundglass screen. Using a chinrest, the subject viewed the silhouettes monocularly with his right eye through an eyepiece containing a lens placed at its focal length from the screen. A 5-diopter collimating lens was placed at its focal length in front of the light source for Condition 1 , the parallel projection condition, and was removed for the four polar conditions. Table 2 shows the distances of the light source and stimuli from the screen, the visual angle (v.a.) subtended by the horizontal stimulus component in the frontal plane, and the values of $\varphi$ for the five conditions. Condition 3 was run as a control to ascertain whether or not an increase in v.a. subtended by the stimulus affected the number of perceived reversals. Due to the nature of the apparatus, it was not possible to equate the values of $\varphi$ for Conditions 3 and 4 . Therefore, $\varphi$ values for Conditions 3 and 4 were very close, while v.a. for Conditions 3 and 5 were identical.

The stimuli were attached to a vertical gear, coupled to a variable-speed motor, by vertical rods which served as the axes of rotation. A cam placed on the gear triggered a microswitch which tripped a counter to record number of revolutions. A pushbutton was provided for the subject which, when depressed and released, caused a signal to be recorded on a tape recorder.

Procedure. The session began by asking the subject to sit down and look through the eyepiece. When the chinrest had been adjusted for comfort, the following instructions were read to the
Table 1

Lengths (in Millimeters) of Long Vertical Sides of Stimulus Figures

\begin{tabular}{cccc}
\hline Stimulus & Length & Stimulus & Length \\
\hline 1 & 25.25 & 6 & 26.00 \\
2 & 25.35 & 7 & 26.25 \\
3 & 25.50 & 8 & 26.50 \\
4 & 25.60 & 9 & 27.50 \\
5 & 25.75 & 10 & 29.00 \\
\hline
\end{tabular}

Table 2

Distances (in Centimeters) of Light Source $\left(d_{1}\right)$ and Stimuli $\left(d_{2}\right)$ From Screen, Visual Angle (va) Subtended by the Horizontal Stimulus Component in the Frontal Plane, and Value of $\varphi$ for Five Conditions

\begin{tabular}{ccrcc}
\hline Condition & $\mathrm{d}_{1}$ & \multicolumn{1}{c}{$\mathrm{d}_{2}$} & va & $\varphi$ \\
\hline 1 & 67.5 & 7.0 & $17^{\circ} 8^{\prime}$ & $0.000^{\circ}$ \\
2 & 67.5 & 7.0 & $19^{\circ} 0^{\prime}$ & $0.083^{\circ}$ \\
3 & 37.5 & 12.0 & $23^{\circ} 32^{\prime}$ & $0.160^{\circ}$ \\
4 & 37.5 & 7.0 & $20^{\circ} 54^{\prime}$ & $0.183^{\circ}$ \\
5 & 25.0 & 7.0 & $23^{\circ} 32^{\prime}$ & $0.355^{\circ}$ \\
\hline
\end{tabular}

subject: "You will see a figure in the center of the screen. Look at the whole figure, not at the sides. When I turn on the motor, the figure will move. It may occasionally change direction, or move back and forth. Your task is to press this button as soon as you see it change direction. Do not confuse slowing down but continuing in the same direction with a change of direction. Do you understand what to do?"

The stimuli were then presented to the subject. Each stimulus figure was started from the same position, and appeared to the subject initially as a vertical bar. Care was taken to avoid sequential effects by the use of a 10 by 10 Latin square design. Twelve trials (complete rotations) were given for each stimulus.

\section{Results}

Figure 1 shows the mean number of perceived reversals as a function of the difference (millimeters) between long and short vertical sides of the 10 stimuli

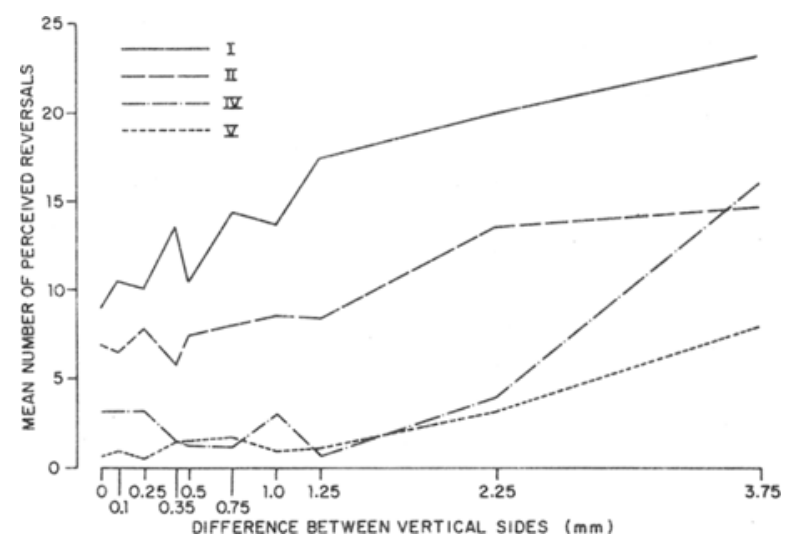

Figure 1. Mean number of perceived reversals as a function of difference between long and short vertical sides (millimeters for 10 stimulus figures for conditions $1,2,4$, and 5 . 
for Conditions $1\left(\varphi=0^{\circ} / \mathrm{sec}\right)$, Condition $2(\varphi=$ $\left..083^{\circ} / \mathrm{sec}\right)$, Condition $4\left(\varphi=.183^{\circ} / \mathrm{sec}\right)$, and Condition $5\left(\varphi=.355^{\circ} / \mathrm{sec}\right)$. As predicted, in all four conditions, the rectangular stimulus oscillated less frequently than the most extreme trapezoidal figure. In addition, an increase in the value of $\varphi$ resulted in a decrease in frequency of perceived oscillations for nearly all stimuli. For Condition 1, the mean frequency of perceived oscillation for Stimulus 1 is not significantly different from $50 \%(\mathrm{t}=1.01$, $\mathrm{p}>.95$ ); it is not significantly different from $100 \%$ for Stimulus $10(\mathrm{t}=1.22, \mathrm{p}>.95)$.

Table 3 shows the standard error of the mean for the 10 stimulus figures for Condition 1 and Condition 5. The standard error (SE) is lowest for Stimulus 10 , the extreme trapezoid, in the parallel projection condition $(\mathrm{SE}=.5)$ and for Stimulus 1 , the rectangle, in the high $\varphi$ condition $(\mathrm{SE}=.4$ ). The $\mathrm{SE}$ increases as the stimuli become more rectangular in Condition 1 and as they become more trapezoidal in Condition 5. Standard errors of the mean showed no patterns for Condition 2,3, and 4.

Figure 2 shows mean number of perceived oscillations as a function of the difference (millimeters) between long and short vertical sides of the stimuli for Condition 3, the control condition $\left(\varphi=.160^{\circ} \%\right.$ sec), Condition $4\left(\varphi=.183^{\circ} / \mathrm{sec}\right)$, and Condition 5 $\left(\varphi=.355^{\circ} / \mathrm{sec}\right)$. For Stimuli 1 through 9 , there is little difference in mean number of perceived reversals between the three conditions. However, the mean number of perceived reversals for Stimulus 10 is very close for Conditions 3 and 4 , where the values of $\varphi$ are similar. The mean number of perceived reversals for Stimulus 10 in Condition 5 , where $\varphi$ was high, is much lower, although v.a. for Conditions 3 and 5 was the same.

An analysis of variance was performed on Conditions $1,2,4$, and 5 . Differences between conditions were found to be significant $(F=22.09$, $\mathrm{p}<.01$ ), differences between the stimuli were found to be significant $(F=4.08, p<.01)$, and the interaction between conditions and stimuli was found to be significant $(F=7.635, p<.01){ }^{1}$

\section{EXPERIMENT 2}

Experiment 2 was conducted to determine the effect of varying speed of rotation on perceived oscillation of the projected stimuli. Speed of rotation was manipulated using both polar and parallel lighting conditions in order to test whether or not a decrease in number of perceived oscillations can be attributed to an increase in the effectiveness of movement parallax at higher speeds.

\section{Method}

Four separate conditions were run. For Conditions 1 and $2, \varphi=.83^{\circ} / \mathrm{sec}$, v.a. equalled $19^{\circ}$; for Conditions 3 and 4 ,
Table 3

Standard Error of the Mean for 10 Stimulus Figures for Conditions 1 and 5

\begin{tabular}{ccc} 
& \multicolumn{2}{c}{ Standard Error } \\
\cline { 2 - 3 } Stimulus & Condition 1 & Condition 5 \\
\hline 1 & 2.0 & .4 \\
2 & 2.7 & 1.6 \\
3 & 2.4 & .3 \\
4 & 1.6 & 1.1 \\
5 & 2.8 & .8 \\
6 & 2.5 & .8 \\
7 & 1.7 & .9 \\
8 & 1.7 & .6 \\
9 & 1.7 & 1.5 \\
10 & .5 & 2.3 \\
\hline
\end{tabular}

Note-For Condition 1, $\varphi=0 ;$ for Condition $5, \varphi=.355$.

$\varphi=0^{\circ} / \mathrm{sec}$, v.a. equaled $17^{\circ} 8^{\prime}$. For Conditions 1 and 3 , speed of rotation was $5 \mathrm{rpm}$; for Conditions 2 and 4 , speed of rotation was $20 \mathrm{rpm}$. Ten different subjects participated in each condition. The apparatus and procedure were identical to those of Experiment 1 .

\section{Results}

Figure 3 shows mean number of perceived reversals as a function of the difference (millimeters) between long and short stimulus sides for Conditions 1 and 2, the polar lighting conditions.

As speed of rotation was increased from 5 to $20 \mathrm{rpm}$, there was a decrease in the mean number of perceived reversals for all stimuli. A $t$ test was conducted on these data, and was found to be significant $(\mathrm{t}=4.75, \mathrm{p}<.01)$.

Figure 4 shows mean number of perceived reversals as a function of the difference (millimeters) between long and short vertical sides of the stimuli for Conditions 3 and 4, the parallel lighting conditions. As speed of rotation was increased, mean number of perceived reversals decreased for all figures. A t test on difference between the means was significant $(\mathrm{t}=6.57, \mathrm{p}<.01)$.

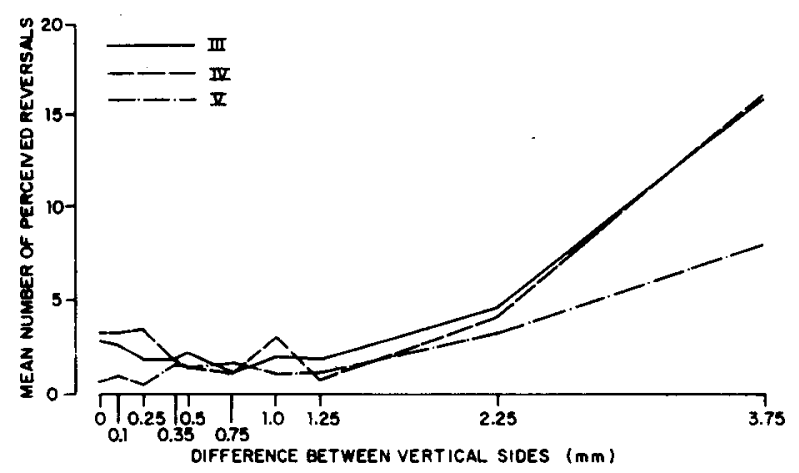

Figure 2. Mean number of perceived reversals as a function of difference between long and short vertical sides (millimeters) for 10 stimulus figures for Conditions 3,4 , and 5 . 


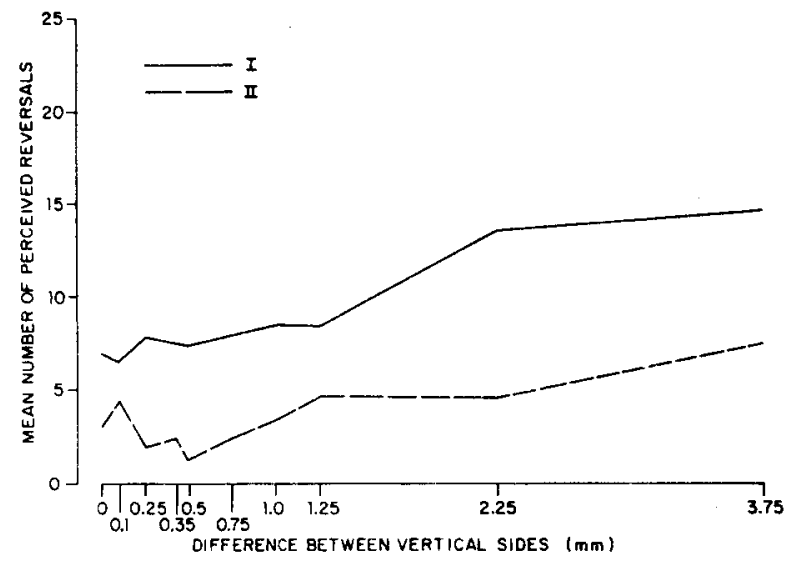

Figure 3. Mean number of perceived reversals as a function of difference between long and short vertical sides (millimeters) for 10 stimulus figures for Conditions 1 and 2.

In comparing Figures 3 and 4, it can be noted that, with speed constant at $5 \mathrm{rpm}$, a decrease in $\varphi$ from $.083^{\circ} / \mathrm{sec}$ to $0^{\circ} / \mathrm{sec}$ (Conditions 1 and 3 ) resulted in an increase in mean number of perceived reversals for all figures, as was found in Experiment 1. However, at $20 \mathrm{rpm}$, an identical decrease in $\varphi$ between Conditions 2 and 4 did not result in a similar increase in the mean number of perceived reversals.

\section{GENERAL DISCUSSION}

The data from Condition 1, Experiment 1, confirm previous findings (Braunstein \& Payne, 1967) that a screen-projected rectangle displayed in parallel projection will be ambiguous (oscillate $50 \%$ of the time), and that a screen-projected trapezoid shown in parallel projection will oscillate $100 \%$ of the time

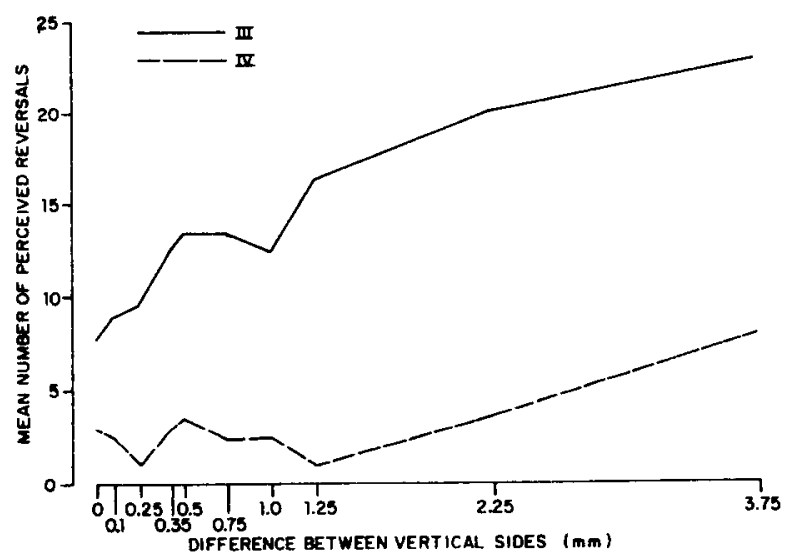

Figure 4. Mean number of perceived reversals as a function of difference between long and short vertical sides (millimeters) for 10 stimulus figures for Conditions 3 and 4 .
(Braunstein \& Payne, 1967; Graham \& Gillam, 1970). Thus, when the cue of movement parallax is ambiguous, the observer relies on the cue of linear perspective in order to obtain information about the direction of rotation of a stimulus. The results of Experiment 1 show further that, when the relative strengths of the two cues are covaried, there is a predictable increase or decrease in the number of perceived reversals, as can be seen from the data of Conditions $1,2,4$, and 5 . The analysis of variance indicated that there was a significant difference between mean number of perceived oscillations for different values of $\varphi$, as well as for different values of linear perspective, i.e., an increase in the strength of linear perspective has been shown to lead to a significant increase in the mean number of perceived oscillations, and an increase in the strength of movement parallax has been shown to lead to a significant decrease in the mean number of perceived oscillations. Since v.a. for Conditions 3 and 5 was identical, the v.a. subtended by the stimulus cannot account for these differences; it is the value of $\varphi$ which is the important variable in determining the number of oscillations perceived. The first hypothesis of Experiment 1 was therefore confirmed.

It was also found in Experiment 1 that there was a significant interaction effect between the two cues. In addition, the data of Table 3 indicate that variability between subjects increases as the ambiguity of the stimulus display increases, as predicted by the second hypothesis of Experiment 1. The implications of these findings are, first, that the two cues may be given different "weightings" by the visual system, depending on their relative strengths in the stimulus situation; and, secondly, that in situations of perceptual conflict, different observers may give different "weights" to one or the other cue, as Zegers and Murray (Note 1) argued.

It should be again noted that Zegers and Murray (Note 1), as well as numerous other investigators, utilized planar objects rotating in space in their experiments concerning the Ames illusion. The data from experiments using rotating objects and from those which employ two-dimensional screen projections of rotating stimuli are in agreement. Therefore, provided the requirements for producing the KDE are met, i.e., changes in both apparent size and direction of the stimuli occur in a projection display (Wallach \& O'Connell, 1953), it would seem that either method of stimulus presentation may be successfully used.

The results of Experiment 2 show that, under both polar and parallel lighting conditions, an increase in speed of rotation of the stimuli leads to a significant decrease in the mean number of perceived 
reversals. Since the cue of movement parallax is ambiguous in parallel projection, this decrease cannot be attributed to an increased effectiveness of this cue at higher rotational speeds. An alternative explanation is that the other cue involved in producing apparent oscillation of the stimulus figure, linear perspective, is better utilized by the visual system at lower speeds of rotation. The above explanation is supported by the finding that, at $20 \mathrm{rpm}$, there is not the same increase in number of perceived reversals when $\varphi$ is decreased that one finds at $5 \mathrm{rpm}$.

\section{REFERENCE NOTE}

1. Zegers, R. T., \& Murray, P. Perception of distortion. [1] An experimental approach to illusion. Navtradevcen 506-1, U.S. Naval Training Device Center, Port Washington, New York, October 1962.

\section{REFERENCES}

Ames, A. Visual perception and the rotating trapezoidal window. Psychological Monographs, 1951, 65, Whole No. 324.

Borjesson, E. Properties of changing patterns evoking visually perceived oscillation. Perception \& Psychophysics, 1971, 9, 303.308.

Borjesson, E., \& Hofsten, C. von. Spatial determinants of depth perception in two-dot motion patterns. Perception \& Psychophysics, 1972, 11. 263-268.

Braunstein, M. L. The perception of depth through motion. Psychological Bulletin, 1962, 59, 422-433.

Braunstein, M. L.. \& Payne J. W. Perspective and the rotating trapezoid. Joumal of the Optical Society of America, 1967. 58. 399-403.

CAHILl. M. Accuracy of position judgments of stationary targets yielded by three types of perceiver of the Ames trapezoidal illusion. Proceedings of the 77 th Annual Convention of the A.P.A., 1969. pp. 29.30.

CoOK. T., Mefferd, R., \& Wieland, B. Apparent reversals of orientation (perspective reversals) in depth as determinants of apparent reversals of rotary motion. Perceptual and Motor Skills, 1967, 24, 691-702.

Cross, J. T. Linear perspective as a cue in misperceived rotary motion. Perception \& Psychophysics, 1969, 6, 145-146.

Cross. J. F., \& Cross, J. The misperception of rotary motion. Perception \& Psychophysics, 1969, 5, 94-96.

DAY, R. H., \& Power, R. P. Apparent reversal (oscillation) of rotary motion in depth: An investigation and a general theory. Psychological Review, 1965, 72, 117-127.
Freeman, R. B., \& Pasnak, R. Perspective determinants of the rotating trapezoid illusion. Joumal of Experimental Psychology, 1968, 76, 94-101.

Gibson, J. J. The senses considered as perceptual systems. Boston, Houghton-Mifflin, 1966.

Gillam, B. Comment on MacRae and Powers "Critical comment on one aspect of Graham's 'On some aspects of real and apparent visuai movement.' "Journal of the Optical Society of America, 1969, 60, 284.

GRAHAM, C. H. On some aspects of real and apparent visual movement. Journal of the Optical Society of America, 1963. 53, 1019-1025.

Graham, C. H., \& Gillam, B. Occurrence of theoretically correct responses during rotation of the Ames window. Perception \& Psychophysics, 1970, 8, 257-260.

Hershberger, W. A. Comments on "Apparent reversal (oscillation) of rotary motion in depth." Psychological Review, 1967, 74, 235-238.

ITtLeson, W. H. Visual space perception. New York: Springer, 1960.

Johanssen, G. Visual perception of biological motion and a model for its analysis. Perception \& Psychophysics, 1973, 14, 201-211.

Kilpatrick, F. The Ames oscillatory effect: A reply to Pastore. Psychological Review, 1953, 60. 76-79.

Mc Nemar, O. Psychological statistics. New York: Harcourt, Brace, 1955.

MEtzer, W. Beobachtungen über phänomenale Identität. Psychologische Forschung, 1934, 19. 1-60.

Mulholiand, T. Motion perceived while viewing rotating stimulus objects. American Journal of Psychology, 1956, 69, 96-99.

Murch, G. M. Perception of rotary movement. Journal of Experimental Psychology, 1970, 86, 83-85.

Pastore. N. Some remarks on the Ames oscillatory effect. Psychological Review, 1952, 59. 319-323.

POwER, R. R. Stimulus properties which reduce apparent reversal of rotating rectangular shapes. Journal of Experimental Psychology, 1967, 73, 595-599.

Wallach, H.. \& O'Connell, D. N. The kinetic depth effect. Journal of Experimental Psychology, 1953, 45, 205-217.

ZEGERs, $R$, T. The reversai illusion of the Ames trapezoid. Translations of the New York Academy of Sciences, 1965, 26, 377.400 .

\section{NOTE}

1. From McNemar (1955, pp. 332-335) Case XVII mixed-model pseudo three-way classification.

(Received for publication July 25, 1977; revision accepted September 2, 1977.) 